\title{
Facturation séparée pour l'assurance de base et l'assurance complémentaire
}

Service tarifaire de la FMH et service juridique de la FMH

Hanspeter Kuhn, avocat, secrétaire général adjoint de la FMH
L'ordonnance sur l'assurance-maladie (OAMal) actuellement en vigueur stipule en son article 59, alinéa 3, que les prestations prises en charge par l'assurance obligatoire des soins doivent être clairement distinguées des autres prestations sur les factures. Cette distinction ne suffira plus à partir $\mathrm{du} 1^{\mathrm{er}}$ janvier 2009. Dans le cadre des modifications apportées à l'OAMal, le Conseil fédéral a décidé le 22 octobre 2008 qu'à partir du $1^{\text {er }}$ janvier 2009 (!), les prestataires de soins devront établir deux factures séparées pour les prestations prises en charge par l'assurance obligatoire des soins et pour les autres prestations. Dans son commentaire sur les modifications de l'ordonnance, l'OFSP indique que les données liées au diagnostic «comprennent des informations considérées comme sensibles au sens de la loi sur la protection des données», et que la révision vise à garantir leur

\section{Commentaire}

Cette modification de l'OAMal n'était pas encore prévue lors de la procédure de consultation. De ce fait, la FMH n'a pas eu la possibilité de s'exprimer à ce sujet dans un premier temps; aujourd'hui, nous sommes amenés à formuler le commentaire suivant:

La modification de l'art. 59 OAMal fait sens pour une petite partie des cas de traitement, précisément lorsque le patient n'est pas couvert par le même assureur pour l'assurance de base et l'assurance complémentaire.

En revanche, si le patient détient ces deux assurances auprès de la même caisse-maladie, on peut supposer qu'il souhaite que seul un nombre aussi restreint que possible de personnes s'occupent de son cas et aient des informations sur sa santé. Par conséquent, il serait plus convaincant de ne pas prescrire deux factures séparées pour ces cas de prestations combinées mais, au contraire, d'étendre les règles relatives au médecin-conseil LAMal à l'assurance complémentaire lorsqu'elle est gérée par la même caisse. Cette mesure est préconisée depuis des années non seulement par la FMH et la Société suisse des médecins-conseils. La Commission d'experts pour la protection de la personnalité dans l'assurance-maladie et accidents sociale et privée, instaurée par la conseil- utilisation exclusive à des fins de contrôle des factures et du caractère économique des prestations. Toujours selon l'Office, le fait que de nombreux assurés ne soient pas assurés auprès du même groupe pour l'assurance de base et l'assurance complémentaire est également pris en compte et, dans la même optique, on a voulu veiller à ce que l'assureur sépare entièrement l'un de l'autre les processus administratifs des deux types d'assurance. Outre la protection des données, l'établissement de factures séparées améliore aussi la transparence.

La FMH a protesté contre la brièveté du délai et l'information tardive de l'OFSP; elle a par ailleurs exigé un délai transitoire suffisant pour permettre, entre autres, une adaption des programmes informatiques des cabinets médicaux.

lère fédérale Ruth Dreifuss et le conseiller fédéral Arnold Koller en 1998 et présidée par le Professeur en droit Thomas Geiser, avait elle aussi proposé cette extension du filtre constitué par le médecin-conseil aux assurances-complémentaires des caisses [1].

L'idée de séparation avancée par le Conseil fédéral s'arrête à mi-chemin: si l'assurance de base et l'assurance complémentaire doivent faire traiter le même cas par des collaborateurs différents dans le cadre de deux administrations séparées, je ne vois plus de raison valable de permettre à une caisse-maladie de gérer l'assurance complémentaire à côté de l'assurance de base. Le législateur devrait faire un pas de plus et séparer ces deux branches d'assurance, à l'instar de l'assurance-vie qui est institutionnellement détachée depuis des décennies des autres assurances de personnes. Par conséquent, il conviendrait de réviser l'art. 12, 2e alinéa LAMal [2] et de lui donner la teneur suivante: «Les caisses-maladies reconnues pratiquent exclusivement l'assurance sociale des soins et l'assurance sociale d'indemnités journalières au sens de la présente loi.»

Le Conseil fédéral fonde la révision de l'OAMal sur la seule LAMal, laquelle règle uniquement l'assurance de base. De ce fait, l'OAMal 
révisée signifie seulement, mais tout de même, que la facture destinée à l'assurance de base ne peut dorénavant contenir que des prestations à prise en charge obligatoire.

Le Code des obligations laisse toute liberté aux mandants et aux mandataires de régler euxmêmes les modalités de paiement des prestations non obligatoires: facture écrite avec délai de paiement de 30 jours? Paiement au comptant ou paiement avec carte ec-direct contre quittance (comme c'est l'usage depuis longtemps pour les médicaments en vente libre achetés en pharmacie)?

\section{Références}

1 Office fédéral des assurances sociales. Protection de la personnalité dans l'assurance-maladie et accidents sociale et privée. Berne: OFAS; 2001. p. 128 et 147. La Commission avait proposé un nouvel alinéa 1a à l'art. 57 LAMal: «Si un assureur offre, en sus de l'assurance-maladie sociale selon la présente loi, des assurances complémentaires ou s'il exploite d'autres assurances, les dispositions relatives aux médecins-conseils s'appliquent également à ces assurances. Déjà lors de la conclusion de l'assurance, il peut être exigé que les données médicales soient accessibles exclusivement au service du médecin-conseil.»

2 L'art. 12, al. 2 a actuellement la teneur suivante: «Les caisses-maladie ont le droit de pratiquer, en plus de l'assurance-maladie sociale au sens de la présente loi, des assurances complémentaires; elles peuvent également pratiquer d'autres branches d'assurance, aux conditions et dans les limites fixées par le Conseil fédéral.»

\section{Convention relative au for judiciaire et à l'élection de droit en anglais}

\author{
Gabriela Lang
}

1 Kunz R, Kuhn HP. Tendances actuelles en matière d'assurance responsabilité civile professionnelle. Les règles à suivre pour le corps médical libéral en Suisse. Bull Méd Suisses. 2006;87(51-52): 2226-7.

Correspondance:

Gabriela Lang, avocate

Service juridique de la FMH

Elfenstrasse 18

Case postale 170

CH-3000 Berne 15

Tél. 0313591111

Fax 0313591112

lex@hin.ch
Une convention sur le for judiciaire et l'élection de droit, rédigée par le Professeur Jürgen Brünnimann, dr en droit et spécialiste en procédure civile de Berne, a été publiée à la page 2227 dans le Bulletin des médecins suisses no 51-52/2006 à l'intention des médecins qui comptent de nombreux patients étrangers - en particulier des Etats-Unis et du Canada - parmi leur clientèle [1]. Depuis lors, les questions posées par le corps médical au service juridique de la FMH ont révélé la nécessité de traduire cette convention en anglais afin que les patients anglophones ne puissent pas arguer du fait qu'ils ne l'ont pas comprise. La version anglaise est la suivante:

- Choice of law: For the legal relationship between the parties, and in particular for all claims related to examinations, treatments and any other services (performed by Dr XY / at Z Hospital), Swiss substantive law, namely the Swiss Code of Obligations, is applicable.

- Place of jurisdiction: The sole place of jurisdiction for all disputes in connection with this agreement is (place), Switzerland. (Dr XY / Z Hospital) is entitled, at his/her own discretion, to place the matter before the ordinary courts at the patient's domicile.
Ce texte peut également être téléchargé sur le site Internet de la FMH (www.fmh.ch $\rightarrow$ Nos prestations $\rightarrow$ Droit $\rightarrow$ Modèles de documents).

La remarque de 2006 concernant cette convention sur le for judiciaire et l'élection de droit conserve sa validité: «Ces conventions sont en principe reconnues et d'usage en vertu du droit suisse et il est recommandé de les appliquer, même s'il subsiste certains risques. En effet, un tribunal, par exemple aux Etats-Unis ou au Canada, pourrait être tenté de ne pas en respecter les dispositions et d'intervenir dans le cadre de l'action introduite par le citoyen américain ou canadien concerné. Le cas échéant, il pourrait alors lui accorder l'une des prétentions à des dommagesintérêts exorbitantes qui sont courantes dans ces pays. Il faut préciser qu'il est peu probable qu'un tel jugement soit exécuté dans le contexte qui prévaut actuellement en Suisse, parce qu'il aura été prononcé en violation du droit de notre pays. Par contre, si vous deviez voyager par la suite aux Etats-Unis ou au Canada ou si vous y aviez constitué des valeurs en capital, une action pécuniaire pourrait quand même être engagée à votre encontre.» 